\title{
Cuatro compuestos nuevos del extracto no polar de la planta Amyris brenesii (Rutaceae) de Costa Rica
}

\author{
Max Chavarría ${ }^{1}$, Victor Castro ${ }^{1}$, Luis Poveda ${ }^{2} \&$ Renato Murillo ${ }^{1}$ \\ 1. Centro de Investigación en Productos Naturales (CIPRONA), Universidad de Costa Rica, San José, Costa Rica; \\ mcharria@equi.ucr.ac.cr; vhcastro@equi.ucr.ac; crrenato@equi.ucr.ac.cr \\ 2. Escuela de Ciencias Ambientales, Facultad de Ciencias de la Tierra y el Mar, Universidad Nacional, Heredia, Costa \\ Rica; lpoveda@una.ac.cr
}

Recibido 14-II-2007. Corregido 30-VI-2008. Aceptado 13-III-2008.

\begin{abstract}
Four new compounds from the non-polar extract of the plant Amyris brenesii (Rutaceae) from Costa Rica. Fractionation of a non polar extract of the aerial parts of Amyris brenesii collected in Río Cuarto, Grecia, Costa Rica has resulted in the isolation of four new compounds, 6-hidroxy-6-O-(3-hidroxymethyl-3methylalyl)-angelicin 1, 6-(N-acetyl-2-etanamin)-2,2-dimethyl-2H-cromen 2, the lignan 2,5-dehidrohinokinin 3 and $\mathrm{N}$-acetyl-O-(geranyl)-tiramine 4 . In addition, we isolated six previously known compounds: the lignans hinokinin 5 and Justicidin E 6, the coumarins scopoletin 7 and marmesin 8, 24-moretenoic acid 9, and the nitrogen compound O-(3,3-dimethylalyl)-halfordinol 10 . All the separations were done with chromatographic techniques and the structures were elucidated by using 1D and 2D NMR techniques. Rev. Biol. Trop. 56 (3): 1043-1051. Epub 2008 September 30.
\end{abstract}

Key words: Rutaceae, Amyris, brenesii, lignans, furocoumarin, cromen.

La familia Rutaceae comprende a nivel mundial unos 150 géneros y alrededor de 900 especies distribuidas principalmente por los trópicos y regiones templadas, particularmente en África del Sur y Australia. En Costa Rica se han reportado 19 géneros según datos del Instituto Nacional de Biodiversidad (INBio, 2005). La familia tiene gran importancia debido a los frutos (cítricos) de muchas de sus especies, así como a la producción de aceites esenciales y sustancias medicinales.

Un género importante de esta familia es el Amyris, el cual ha generado mucho interés entre los fitoquímicos por la variedad de compuestos que presentan (Hasbun-Pacheco y Castro-Castillo 1988). Por ejemplo de la especie Amyris plumieri se aislaron un grupo de nicotinamidas (Basil y Parkins 1978), de A. simplicifolia (Cordova y Garelli 1974), A. madrensis (Domínguez et al. 1977), A. marshii (Dominguez et al. 1985) y A. barbata (Hasbun-Pacheco y Castro-Castillo 1986, Hasbun-Pacheco y Calderón 1990) una serie de cumarinas y piranocumarinas, y de $A$. brenesii las conocidas cumarinas marmesina y xantiletina, además de compuestos nitrogenados como el O-(3,3-dimetilalil)-halfordinol, el 2-(3'-piridil)-5-(3",4"metilendioxi)-5"'-metoxioxazol y la p-O-(3,3-dimetilalil)-benzamida (Hasbun-Pacheco y Castro-Castillo 1988). Además de la variedad de compuestos, en este género existen un número importante de especies con actividad biológica: la $A$. brenesii presenta actividad antifúngica (Hasbun-Pacheco y Caderón 1990, Hasbun-Pacheco y CastroCastillo 1988), la A. pinnata presenta propiedades citotóxicas (Seida 1979) y en medicina popular se han utilizado especies como la $A$. barbata en el tratamiento de enfermedades en la piel (Holdridge y Poveda 1985). 
En el presente trabajo se reportan los resultados en un nuevo estudio fitoquímico de una muestra de $A$. brenesii recolectada en Costa Rica.

\section{MATERIALES Y MÉTODOS}

Equipo y materiales: las separaciones primarias se realizaron con Silica Gel (60 a 200 $\mu \mathrm{m})$ Merck y Silica Gel (40 a $60 \mu \mathrm{m})$ Merck para la separación en columna con presión media. Los platos cromatográficos (TLC1) se prepararon con una mezcla 1:1 de gel de sílice $60 \mathrm{~F}_{254}$ Merck y yeso. Para la separación de fracciones con masas menores se utilizaron cromatofolios de gel de sílice 20×20 cm gel de sílice $60 \mathrm{~F}_{254}$ Merck (TLC2). El control de las separaciones se llevó a cabo mediante radiación ultravioleta (lámpara UV marca Vpland, UVLS-28) y Resonancia Magnética Nuclear ( $\left.{ }^{1} \mathrm{H}-\mathrm{RMN}\right)$.

La elucidación estructural de los compuestos puros se llevó a cabo con espectros de una y dos dimensiones obtenidos con un Espectrómetro de Resonancia Magnética Nuclear RMN de $400 \mathrm{MHz}$ Varian Mercury 400, a saber, ${ }^{1} \mathrm{H}-\mathrm{RMN},{ }^{13} \mathrm{C}-\mathrm{RMN}$, DEPT, $\mathrm{H}, \mathrm{H}-\mathrm{COSY}$, gHSQC, gHMBC y NOE (Efecto Nuclear Overhauser).

Material vegetal: las partes aéreas de la planta se recolectaron en Río Cuarto, Grecia, Costa Rica en Febrero del año 2002. Se conserva una muestra de esta planta en el Herbario Juvenal Valerio de la Universidad Nacional, número 9154.

Extracción y aislamiento: la planta se secó a temperatura ambiente y se molió obteniéndose una masa de $1000 \mathrm{~g}$ de hojas y $850 \mathrm{~g}$ de troncos. Posteriormente, se maceró de forma separada por $24 \mathrm{~h}$ con una mezcla de éter tertbutilmetílico (MTBE) y metanol $(\mathrm{MeOH})$ en una proporción 9:1.

Extracto obtenido de las hojas: se desengrasó el extracto con metanol a una temperatura de $-20{ }^{\circ} \mathrm{C}$ obteniéndose una masa de $11.8 \mathrm{~g}$. La masa del extracto se fraccionó en una columna (CC) de gel de sílice $(60-200 \mu \mathrm{m})$ utilizando como fase móvil mezclas de disolventes secos con polaridad creciente. Se obtuvieron cinco fracciones: Fr1 hexano:MTBE 85:15, Fr2 hexano:MTBE 65:35, Fr3 hexano:MTBE 40:60, Fr4 MTBE 100\%, Fr5 MTBE: MeOH $85: 15$.

La fracción Fr2 se separó utilizando una columna de presión media utilizando como fase móvil mezclas de disolventes secos con polaridad creciente (hexano:éter 95:5, hexano:éter $85: 15$, hexano:éter 75:25, hexano:éter 50:50, éter $100 \%$ ). De esta separación se obtuvieron siete fracciones (Fr21-Fr27) de las cuales se conservaron las fracciones Fr26 y Fr27. La fracción Fr26 se separó con fase reversa obteniéndose cinco fracciones (Fr261-Fr265) de las cuales se conservan las fracciones Fr261 y Fr265. La fracción Fr261 se separó en TLC2 con $\mathrm{CHCl}_{3}$ :benceno 8:2 obteniéndose el compuesto 5. El compuesto 5 fue purificado en TLC2 con benceno. La fracción Fr265 se separó en TLC1 con benceno: $\mathrm{CH}_{2} \mathrm{Cl}_{2}$ :éter etílico 4,5:4,5:1 obteniéndose el compuesto 9 de forma pura. La fracción Fr27 se separó en TLC1 con benceno: $\mathrm{CH}_{2} \mathrm{Cl}_{2}$ :acetato de etilo 4,5:4,5:1 obteniéndose tres fracciones (Fr271Fr273). La fracción Fr272 se separó en TLC2 con benceno: $\mathrm{CH}_{2} \mathrm{Cl}_{2}$ :éter etílico 4,5:4,5:1 obteniéndose el compuesto 10 .

Por otro lado, la fracción Fr3 se separó utilizando una columna de presión media utilizando como fase móvil mezclas de disolventes secos con polaridad creciente (hexano:éter 95:5, hexano:éter 85:15, hexano:éter 75:25, hexano:éter 50:50, éter $100 \%$ ). De esta separación se obtuvieron ocho fracciones (Fr31-Fr38) de las cuales se conservaron las fracciones Fr32, Fr33, Fr36 y Fr38. Las fracciones Fr32 y Fr36 precipitaron a $-20{ }^{\circ} \mathrm{C}$ obteniéndose los compuestos 6 y un derivado de ácido benzoico. La fracción Fr33 se separó en TLC1 con tolueno:MTBE 95:5 obteniéndose dos fracciones (Fr331 y Fr332). La fracción Fr332 corresponde al compuesto 3 el cual se purificó en TLC2 con tolueno:MTBE 8:2. La Fracción Fr38 se separó en TLC1 con éter:hexano 8:2 
obteniéndose tres fracciones (Fr381-Fr383) de las cuales la fracción Fr383 correspondía al compuesto 4. Finalmente, la fracción Fr382 se separó en TLC2 con éter:hexano 6:2 obteniéndose el compuesto 1.

Extracto obtenido de los troncos: se desengrasó el extracto con metanol a una temperatura de $-20{ }^{\circ} \mathrm{C}$ obteniéndose una masa de 11.5 g. La masa del extracto se disolvió en cloroformo, y se fraccionó en una columna (CC) de gel de sílice grueso $(60-200 \mu \mathrm{m})$ utilizando como fase móvil mezclas de disolventes secos con polaridad creciente. Se obtuvieron cinco fracciones: FrT1 hexano:MTBE 85:15, FrT2 hexano:MTBE 65:35, FrT3 hexano:MTBE 40:60, FrT4 MTBE $100 \%$, FrT5 MTBE:MeOH 85:15. La fracción FrT4 se separó en TLC1 con $\mathrm{CH}_{2} \mathrm{Cl}_{2}$ :acetato de etilo 9:1 obteniéndose cinco fracciones (FrT41-FrT45). En la fracción FrT44 se encontraba el compuesto 8 de forma pura y la fracción FrT43 se separó en TLC2 con $\mathrm{CH}_{2} \mathrm{Cl}_{2}$ :acetato de etilo 9:1 obteniéndose el compuesto 7. La fracción FrT5 se separó en TLC1 con $\mathrm{CH}_{2} \mathrm{Cl}_{2}$ :acetato de etilo 9:1 obteniéndose seis fracciones (FrT51-FrT56). La fracción FrT55 se separó en TLC2 con $\mathrm{CH}_{2} \mathrm{Cl}_{2}$ :acetato de etilo 95:5 obteniéndose el compuesto 2 .

\section{RESULTADOS}

Del estudio fitoquímico de las partes aéreas de Amyris brenesii se obtuvo un total de diez compuestos puros de los cuales 1, 2, 3 y 4 son de estructura novedosa.

Compuesto 1: 6-hidroxi-6-O-(3-hidroximetil-3-metilalil)-angelicina. $\mathrm{C}_{16} \mathrm{H}_{14} \mathrm{O}_{4}$ : $270.29 \mathrm{~g} / \mathrm{mol}$. Compuesto de estructura novedosa, $30 \mathrm{mg}$. $\mathrm{Rf}=0.46 \mathrm{CH}_{2} \mathrm{Cl}_{2}$ : benceno:éter etílico 4.5:4.5:1 (TLC2).

Compuesto 2: 6-(N-acetil-2-etanamin)2,2-dimetil-2H-cromeno. $\mathrm{C}_{15} \mathrm{H}_{19} \mathrm{NO}_{2} 245.32$ $\mathrm{g} / \mathrm{mol}$. Compuesto de estructura novedosa, $10 \mathrm{mg}$. $\mathrm{Rf}=0.54 \mathrm{CH}_{2} \mathrm{Cl}_{2}$ :acetato de etilo 95:5 (TLC2).

Compuesto 3: 2,5-deshidrohinokinina. $\mathrm{C}_{20} \mathrm{H}_{16} \mathrm{O}_{6} 352.35 \mathrm{~g} / \mathrm{mol}$. Compuesto de estructura novedosa, $35 \mathrm{mg}$. $\mathrm{Rf}=0.37$ benceno

(TLC2).

Compuesto 4: N-acetil-O-(geranil)tiramina. $\mathrm{C}_{20} \mathrm{H}_{29} \mathrm{NO}_{2} 315.46 \mathrm{~g} / \mathrm{mol}$. Compuesto

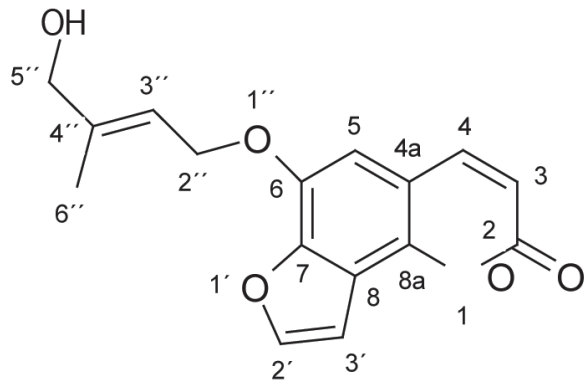

Fig. 1. Estructura de la 6-hidroxi-6-O-(3-hidroximetil-3metilalil)-angelicina 1.

Fig. 1. Structure of 6-hidroxy-6-O-(3-hidroxymethyl-3methylalyl)-angelicin 1 .

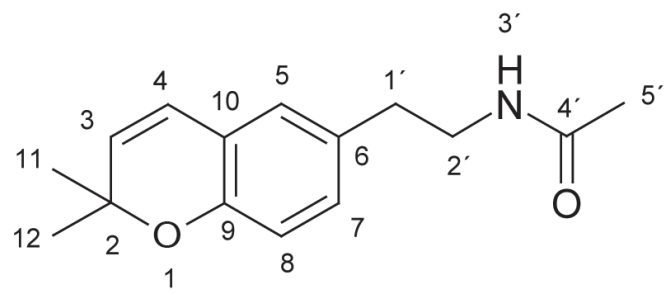

Fig. 2. Estructura del 6-(N-acetil-2-etanamin)-2,2-dimetil$2 \mathrm{H}$-cromeno 2.

Fig. 2. Structure of 6-(N-acetyl-2-etanamin)-2,2-dimethyl$2 \mathrm{H}$-cromen 2

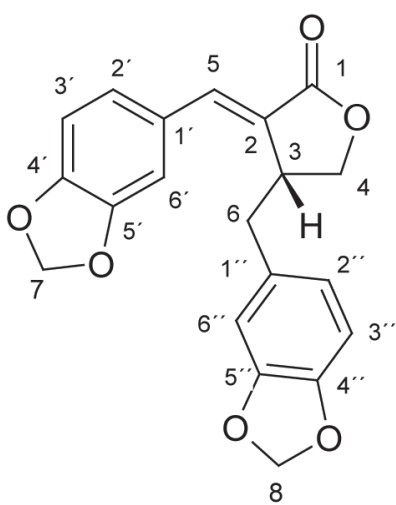

Fig. 3. Estructura de la 2,5-deshidrohinokinina 3.

Fig. 3. Structure of 2,5-dehidrohinokinin 3. 


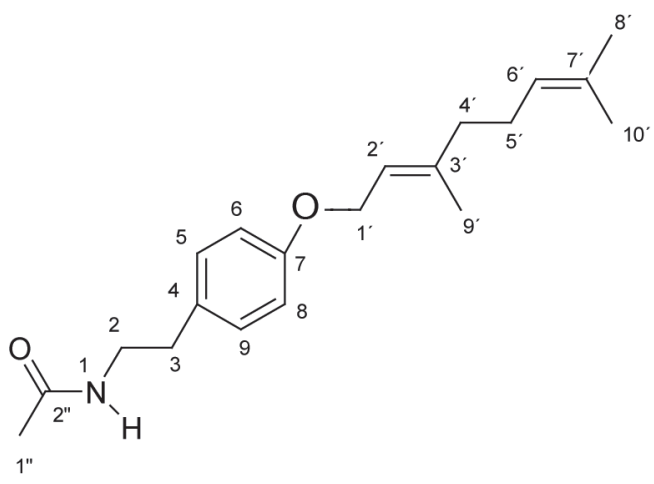

Fig. 4. Estructura de la N-acetil-O-(geranil)-tiramina 4.

Fig. 4. Structure of N-acetyl-O-(geranyl)-tiramine 4.

de estructura novedosa, $50 \mathrm{mg}$. $\mathrm{Rf}=0.13$ éter etílico:hexano 7:3 (TLC2).

Compuesto 5: Hinokinina. $\mathrm{C}_{20} \mathrm{H}_{18} \mathrm{O}_{6}$ $354.36 \mathrm{~g} / \mathrm{mol}$. Compuesto de estructura conocida (Tada y Nakatsuka 1968, Kato et al. 1990), $135 \mathrm{mg}$. Rf=0.71 $\mathrm{CHCl}_{3}$ :benceno 7:3 (TLC2).
Compuesto 6: Justicidina E. $\mathrm{C}_{20} \mathrm{H}_{12} \mathrm{O}_{6}$ $348.32 \mathrm{~g} / \mathrm{mol}$. Compuesto de estructura conocida (Shen et al. 2004, Therien et al. 1993, Subbaraju y Pillai 1996), $41 \mathrm{mg}$. Rf $=0.77$ $\mathrm{CH}_{2} \mathrm{Cl}_{2}$ : benceno:éter etílico 4.5:4.5:1 (TLC2).

Compuestos 7: Escopoletina. $\mathrm{C}_{10} \mathrm{H}_{8} \mathrm{O}_{4}$ $192.19 \mathrm{~g} / \mathrm{mol}$ Compuesto de estructura conocida (Hasbun-Pacheco y Calderón 1990), 10 mg. $\mathrm{Rf}=0.69 \mathrm{CH}_{2} \mathrm{Cl}_{2}$ :acetato de etilo 9:1 (TLC2).

Compuesto 8: Marmesina. $\mathrm{C}_{14} \mathrm{H}_{14} \mathrm{O}_{4}$ $246.27 \mathrm{~g} / \mathrm{mol}$. Compuesto de estructura conocida (Hasbun-Pacheco y Castro-Castillo 1988), $100 \mathrm{mg}$. $\mathrm{Rf}=0.33 \mathrm{CH}_{2} \mathrm{Cl}_{2}$ (TLC2).

Compuesto 9: Acido 24-moretenoico. $\mathrm{C}_{30} \mathrm{H}_{48} \mathrm{O}_{3} 456.72 \mathrm{~g} / \mathrm{mol}$. Compuesto de estructura conocida (Hwang et al. 2003), $30 \mathrm{mg}$. $\mathrm{Rf}=0.58 \mathrm{CHCl}_{3}$ :benceno 7:3 (TLC2).

Compuesto 10: O-(3-3-dimetilalil)halfordinol. $\mathrm{C}_{19} \mathrm{H}_{18} \mathrm{~N}_{2} \mathrm{O}_{2}: 306.37 \mathrm{~g} / \mathrm{mol}$. Compuesto de estructura conocida (HasbunPacheco y Castro-Castillo 1988), $30 \mathrm{mg}$. $\mathrm{Rf}=0.44$ $\mathrm{CH}_{2} \mathrm{Cl}_{2}$ : benceno:éter etílico 4.5:4.5:1 (TLC2).<smiles>CC[C@H](Cc1ccc2c(c1)OCO2)[C@@H](Cc1ccc2c(c1)OCO2)C(C)=O</smiles>

5<smiles></smiles>

6<smiles>COc1cc2ccc(=O)oc2cc1O</smiles><smiles>CC(C)(O)[C@H]1Cc2cc3ccc(=O)oc3cc2O1</smiles>

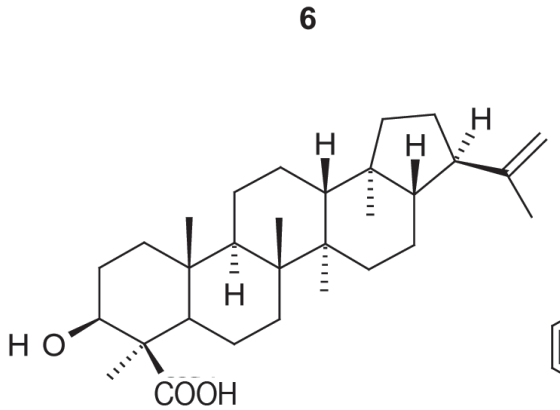

9

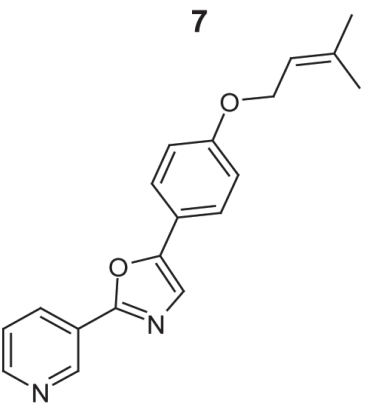

10

Fig. 5. Estructura de los compuestos 5-10 aislados de Amyris brenesii.

Fig. 5. Structures of compounds 5-10 isolated of Amyris brenesii. 


\section{DISCUSIÓN}

De los extractos de MTBE: Metanol 9:1 de las partes aéreas de $A$. brenesii (hojas y troncos) se lograron aislar cuatro compuestos que no han sido reportados en la literatura. La elucidación estructural se realizó con la ayuda de técnicas espectroscópicas de Resonancia Magnética Nuclear (RMN) de una y dos dimensiones, a saber, ${ }^{1} \mathrm{H}-\mathrm{RMN},{ }^{13} \mathrm{C}-\mathrm{RMN}$, DEPT, H,H-COSY, gHSQC, gHMBC y NOE (Efecto Nuclear Overhauser) para la determinación de la estereoquímica de los compuestos.

Los datos espectrales de ${ }^{1} \mathrm{H}-\mathrm{RMN}$ de 1 muestran desplazamientos químicos y multiplicidades que concuerdan con la estructura de una cumarina del tipo de la escopoletina (Cuadro 1). Sin embargo, uno de los singuletes aromáticos no se encuentra presente, sugiriendo así un nuevo patrón de sustitución. De los espectros de desacoplamiento homo- y heteronuclear se pudo deducir la presencia de un hidroximetilmetilalilo enlazado en O-6. La estereoquímica del doble enlace del sistema de isopreno se plantea $Z$ por comparación con sistemas análogos (Kalinowski 1984). Además es posible ver del gHSQC dos carbonos ( $\delta 106.8 \mathrm{~d}$ y $\delta 146.8$ d) y dos hidrógenos $(\delta 6.83 \mathrm{~d}$ y $\delta 7.70 \mathrm{~d})$ que acoplan entre sí con una constante de acoplamiento de $2 \mathrm{~Hz}$; este sistema de acoplamiento y los respectivos cambios químicos es típico de los anillos de furano (Murillo y Jakupovic 2000). En base a estos planteamientos se puede concluir que el compuesto 1 corresponde a una furocumarina angular con estructura análoga a la angelicina pero con un patrón de sustitución hasta ahora no reportado en O-6.

\section{CUADRO 1 \\ Señales de ${ }^{1} \mathrm{H}$-NMR $y{ }^{13} \mathrm{C}$-NMR (400 $\mathrm{MHz}, \mathrm{CDCl}_{3}$ ) del compuesto 1}

TABLE 1

${ }^{1} \mathrm{H}-\mathrm{NMR}$ and ${ }^{13} \mathrm{C}-\mathrm{NMR}$ data $\left(400 \mathrm{MHz}, \mathrm{CDCl}_{3}\right)$ for 1

$\begin{array}{ccccccc}\mathrm{H} & \mathrm{\delta H} / \mathrm{ppm} & \mathrm{m} & \mathrm{J} / \mathrm{Hz} & \mathrm{C} & \delta / \mathrm{ppm} & \mathrm{m} \\ 3 & 6.38 & \mathrm{~d} & 9.6 & 2 & 160.0 & \mathrm{~s} \\ 4 & 7.77 & \mathrm{~d} & 9.1 & 3 & 114.3 & \mathrm{~d} \\ 5 & 7.38 & \mathrm{~s} & -- & 4 & 144.5 & \mathrm{~d} \\ 2^{\prime} & 7.70 & \mathrm{~d} & 2 & 4 \mathrm{a} & 116.5 & \mathrm{~s} \\ 3^{\prime} & 6.83 & \mathrm{~d} & 2 & 5 & 113.5 & \mathrm{~d} \\ 2^{\prime \prime} & 5.03 & \text { brd } & 7.6 & 6 & -- & -- \\ 3^{\prime \prime} & 5.74 & \text { brdd } & 7.6,7.6 & 7 & -- & -- \\ 5^{\prime \prime} & 4.27 & \text { brs } & -- & 8 & -- & -- \\ 6^{\prime \prime} & 1.88 & \text { brs } & -- & 8 \mathrm{a} & -- & -- \\ & & & & 2^{\prime} & 146.8 & \mathrm{~d} \\ & & & & 3^{\prime} & 106.8 & \mathrm{~d} \\ & & & & 2^{\prime \prime} & 68.1 & \mathrm{t} \\ & & & & 3^{\prime \prime} & 122.1 & \mathrm{~d} \\ & & & & 5^{\prime \prime} & 61.8 & \mathrm{t} \\ & & & & 6^{\prime \prime} & 21.4 & \mathrm{q}\end{array}$

Abreviaturas: $\mathrm{m}=$ multiplicidad, $\mathrm{s}=$ singulete, $\mathrm{d}=$ doblete, $\mathrm{t}=$ triplete, brd=doblete ancho, brdd=doblete dobletes ancho, brs=singulete ancho, $\mathrm{q}=$ cuarteto. 
CUADRO 2

Señales de ${ }^{l} \mathrm{H}$-NMR $y{ }^{13} \mathrm{C}$-NMR $\left(400 \mathrm{MHz}, \mathrm{CDCl}_{3}\right)$ del compuesto 2

TABLE 2

${ }^{1} \mathrm{H}$-NMR and ${ }^{13} \mathrm{C}-\mathrm{NMR}$ data $\left(400 \mathrm{MHz}, \mathrm{CDCl}_{3}\right)$ for 2

$\begin{array}{ccccccc}\mathrm{H} & \mathrm{\delta H} / \mathrm{ppm} & \mathrm{m} & \mathrm{J} / \mathrm{Hz} & \mathrm{C} & \delta / \mathrm{ppm} & \mathrm{m} \\ 3 & 5.60 & \mathrm{~d} & 9.6 & 2 & 28.0 & \mathrm{~s} \\ 4 & 6.29 & \mathrm{~d} & 9.6 & 3 & 131.1 & \mathrm{~d} \\ 5 & 6.80 & \mathrm{~d} & 2 & 4 & 122.1 & \mathrm{~d} \\ 7 & 6.90 & \mathrm{dd} & 8,2 & 5 & 126.5 & \mathrm{~d} \\ 8 & 6.70 & \mathrm{~d} & 8 & 6 & 130.8 & \mathrm{~s} \\ 11 & 1.42 & \mathrm{~s} & -- & 7 & 129.2 & \mathrm{~d} \\ 12 & 1.42 & \mathrm{~s} & -- & 8 & 116.4 & \mathrm{~d} \\ 1 & 2.70 & \mathrm{t} & 6.8,6.8 & 9 & 121.4 & \mathrm{~s} \\ 2 & 3.46 & \mathrm{dd} & 13,6.8 & 10 & 151.7 & \mathrm{~s} \\ 3 & 5.4 & \mathrm{brs} & -- & 1 & 39.7 & \mathrm{t} \\ 5^{\prime} & 1.94 & \mathrm{~s} & & 2 & 40.7 & \mathrm{t} \\ & & & & 5 & 170.1 & \mathrm{~s} \\ & & & & 5 & 23.3 & \mathrm{q}\end{array}$

Abreviaturas: $\mathrm{m}=$ multiplicidad, $\mathrm{s}=$ singulete, $\mathrm{d}=$ doblete, $\mathrm{dd}=$ doblete de dobletes, $\mathrm{t}=$ triplete, brs=singulete ancho, $\mathrm{q}=$ cuarteto.

Del espectro ${ }^{1} \mathrm{H}-\mathrm{RMN}, \mathrm{H}, \mathrm{H}-\mathrm{COSY}$ y gHSQC de 2 se puede deducir la presencia de un anillo aromático 1,2,4 trisustituido, un doble enlace cis, un sistema $\mathrm{CH}_{2} \mathrm{CH}_{2} \mathrm{NH}$ y un grupo acetilo. Por correlaciones del $\mathrm{CH}_{2}$ a $\delta 2.70 \mathrm{t}$ con tres señales del anillo aromático a $\delta 130.8 \mathrm{~s}, \delta 129.2 \mathrm{~d}$ y $\delta 126.5 \mathrm{~d}$ se deduce la presencia de un derivado del fragmento $\mathrm{C}_{6} \mathrm{C}_{2} \mathrm{~N}$; del espectro gHMBC se deriva que el $\mathrm{NH}$ se encuentra acetilado. Los cinco carbonos restantes sugieren la presencia de un prenilo; los dos metilos (singulete a $\delta$ 1.42) presentan correlaciones $J^{2}$ y $J^{3}$ con $\delta 76.2 \mathrm{~s}$ y $\delta 131.1 \mathrm{~d}$ (uno de los carbonos del doble enlace cis a $J^{1}$ con $\delta 5.60 \mathrm{~d}$ ) y el hidrógeno restante del doble enlace cis presenta correlaciones en el gHMBC con las señales del anillo aromático a $\delta 121.4 \mathrm{~s}, \delta 126.5 \mathrm{~d}$ y $\delta 151.6 \mathrm{~s}$. Estas últimas observaciones concuerdan con el sistema de cromona planteado para el compuesto 2. Las correlaciones restantes en el gHMBC coinciden con la estructura indicada.

De los espectros ${ }^{1} \mathrm{H}-\mathrm{RMN}$ y ${ }^{13} \mathrm{C}-\mathrm{RMN}$ de 3 y de las correlaciones $J^{1}$ obtenidas del experimento gHSQC puede detectarse para este compuesto la presencia de dos anillos aromáticos 1,3,4 trisustituidos, seis carbonos y la presencia de dos singuletes anchos en ${ }^{1} \mathrm{H}-\mathrm{RMN}$ (que integran para dos hidrógenos cada uno) a $\delta 5.94$ y $\delta 6.04$ y que correlacionan en $J^{1}$ respectivamente con carbonos a $\delta 101.0$ t y 101.7 t sugieren la presencia de dos dioxometilenos. Estas observaciones sugieren que el compuesto se trata de un lignano. Del experimento gHMBC se observa que H-5 ( $\delta 7.50 \mathrm{~d})$ presenta correlaciones heteronucleares a larga distancia con carbonos aromáticos $(\delta 126.1 \mathrm{~d}$, $\delta 108.7 \mathrm{~d})$ y además con $\mathrm{C}-1$ ( $\delta 172.5 \mathrm{~s})$, se sugiere que en este fragmento del fenilpropanoide el carbono $\mathrm{C}-1$ se encuentra oxidado a 
carboxilato. Al presentar las señales de $\mathrm{H}-4$, $\delta 4.26 \mathrm{~m}$ correlaciones $J^{3}$ con $\mathrm{C}-1$ y con C-6 (Cuadro 3), y al presentar H-6 correlaciones heteronucleares a larga distancia con tres carbonos del otro anillo aromático $(\delta 131.5 \mathrm{~s}, \delta$ $122.1 \mathrm{~d}$ y $\delta 109.2 \mathrm{~d}$ ), se pueden identificar no solo la secuencia del otro fenilpropanoide, sino también el tipo de acoplamiento que se dio entre las dos unidades $\mathrm{C}_{6} \mathrm{C}_{3}$, a saber, del tipo del matairesinol. Además, se puede observar que los cambios químicos de los hidrógenos de uno de los anillos aromáticos se encuentran desplazados a campo más bajo con respecto al otro. La presencia de un doble enlace entre C2 y C5 es la causante del desplazamiento a campo bajo de las señales de uno de los anillos aromáticos, además, el desplazamiento de H-5 a $\delta 7.50 \mathrm{~d}$ concuerda con su posición paralela al carbonilo y además bencílica. El compuesto posee una raíz carbonada análoga al conocido lignano hinokinina el cual también fue aislado en este trabajo (compuesto 5) pero presenta un doble enlace entre los carbonos 2 y 5 (Fig. 3). Este compuesto es un derivado de la hinokinina que no ha sido descrito en la literatura hasta el momento.

CUADRO 3

Señales de ${ }^{1} \mathrm{H}$-NMR $y{ }^{13} \mathrm{C}-\mathrm{NMR}\left(400 \mathrm{MHz}, \mathrm{CDCl}_{3}\right)$ del compuesto 3

TABLE 3

${ }^{1} \mathrm{H}-\mathrm{NMR}$ and ${ }^{13} \mathrm{C}-\mathrm{NMR}$ data $\left(400 \mathrm{MHz}, \mathrm{CDCl}_{3}\right)$ for 3

\begin{tabular}{|c|c|c|c|c|c|}
\hline $\mathrm{H}$ & $\delta \mathrm{H} / \mathrm{ppm}$ & $\mathrm{m}$ & $\mathrm{J} / \mathrm{Hz}$ & $\mathrm{C}$ & $\delta / \mathrm{ppm}$ \\
\hline 2 & -- & -- & -- & 1 & 172.5 \\
\hline 3 & 3.74 & $\mathrm{~m}$ & -- & 2 & 125.8 \\
\hline $4 \alpha$ & \multirow{2}{*}{4.26} & \multirow{2}{*}{$\mathrm{m}$} & \multirow{2}{*}{--} & 3 & 39.9 \\
\hline $4 \beta$ & & & & 4 & 69.5 \\
\hline 5 & 7.50 & d & 2 & 5 & 137.3 \\
\hline $6 \alpha$ & 2.99 & $\mathrm{dd}$ & $17,4.4$ & 6 & 37.6 \\
\hline $6 \beta$ & 2.58 & $\mathrm{dd}$ & 17,10 & $1^{\prime}$ & 128.2 \\
\hline $2^{\prime}$ & 7.08 & $\mathrm{dd}$ & $8,1.6$ & 2 & 126.1 \\
\hline 3 & 6.88 & $\mathrm{~d}$ & 8 & 3 & 108.8 \\
\hline 6 & 7.08 & d & 1.6 & 4 & 149.2 \\
\hline $2 "$ & 6.64 & $\mathrm{dd}$ & $8,1.6$ & 5 & 148.4 \\
\hline 3" & 6.74 & d & 8 & 6 & 108.7 \\
\hline $6 "$ & 6.67 & d & 1.6 & $1 "$ & 131.5 \\
\hline 7 & 6.04 & $\mathrm{~s}$ & -- & $2 "$ & 122.1 \\
\hline \multirow[t]{6}{*}{8} & 5.94 & $\mathrm{~s}$ & -- & $3 "$ & 108.5 \\
\hline & & & & $4 "$ & 146.6 \\
\hline & & & & $5 "$ & 148.0 \\
\hline & & & & $6 "$ & 109.2 \\
\hline & & & & 7 & 101.7 \\
\hline & & & & 8 & 101.0 \\
\hline
\end{tabular}

Abreviaturas: $\mathrm{m}=$ multiplicidad, $\mathrm{s}=$ singulete, $\mathrm{d}=$ doblete, $\mathrm{dd}=$ doblete de dobletes, $\mathrm{t}=$ triplete . 
CUADRO 4

Señales de ${ }^{1} \mathrm{H}$-NMR $y{ }^{13} \mathrm{C}$-NMR $\left(400 \mathrm{MHz}, \mathrm{CDCl}_{3}\right)$ del compuesto 4

TABLE 4

${ }^{1} \mathrm{H}-\mathrm{NMR}$ and ${ }^{13} \mathrm{C}-\mathrm{NMR}$ data $\left(400 \mathrm{MHz}, \mathrm{CDCl}_{3}\right)$ for 4

\begin{tabular}{|c|c|c|c|c|c|c|}
\hline $\mathrm{H}$ & $\delta \mathrm{H} / \mathrm{ppm}$ & $\mathrm{m}$ & $J / \mathrm{Hz}$ & $\mathrm{C}$ & d/ppm & $\mathrm{m}$ \\
\hline 1 & 5.68 & brs & -- & 2 & 40.8 & $\mathrm{t}$ \\
\hline 2 & 3.45 & $\mathrm{dd}$ & $13,6.8$ & 3 & 34.6 & $\mathrm{t}$ \\
\hline 3 & 2.73 & $\mathrm{t}$ & 6.8 & 4 & 130.6 & $\mathrm{~s}$ \\
\hline 5 & 7.08 & $\mathrm{AA}^{\prime} \mathrm{BB}^{\prime}$ & $6.6,2$ & 5 & 129.5 & $\mathrm{~d}$ \\
\hline 6 & 6.85 & $\mathrm{AA}^{\prime} \mathrm{BB}^{\prime}$ & $6.6,2$ & 6 & 114.8 & d \\
\hline 8 & 6.85 & $\mathrm{AA}^{\prime} \mathrm{BB}^{\prime}$ & $6.6,2$ & 7 & 157.5 & $\mathrm{~s}$ \\
\hline 9 & 7.08 & $\mathrm{AA}^{\prime} \mathrm{BB}^{\prime}$ & $6.6,2$ & 8 & 114.8 & d \\
\hline 1 & 4.50 & d & 6 & 9 & 129.5 & d \\
\hline 2 & 5.47 & $\mathrm{t}$ & 6 & 1 & 64.8 & $\mathrm{t}$ \\
\hline 4 & 2.08 & $\mathrm{~m}$ & -- & 2 & 119.4 & d \\
\hline 5 & 2.065 & $\mathrm{~m}$ & -- & 3 & 141.1 & $\mathrm{~s}$ \\
\hline $6^{\prime}$ & 5.08 & $\mathrm{t}$ & 5.8 & 4 & 39.5 & $\mathrm{t}$ \\
\hline 8 & 1.66 & brs & -- & 5 & 26.2 & $\mathrm{t}$ \\
\hline $9^{\prime}$ & 1.72 & $\mathrm{~s}$ & -- & 6 & 123.7 & d \\
\hline 10 & 1.59 & $\mathrm{~s}$ & -- & 7 & 131.7 & $\mathrm{~s}$ \\
\hline \multirow[t]{5}{*}{$1 "$} & 1.92 & $\mathrm{~s}$ & -- & $8^{\circ}$ & 25.6 & $\mathrm{q}$ \\
\hline & & & & 9 . & 16.2 & q \\
\hline & & & & $10^{\circ}$ & 17.6 & q \\
\hline & & & & $1 "$ & 23.2 & q \\
\hline & & & & $2 "$ & 170.2 & $\mathrm{~s}$ \\
\hline
\end{tabular}

Abreviaturas: $\mathrm{m}=$ multiplicidad, $\mathrm{s}=$ singulete, $\mathrm{d}=$ doblete, $\mathrm{dd}=$ doblete de dobletes, $\mathrm{t}=$ triplete, brs $=$ singulete ancho, $\mathrm{q}=$ cuarteto.

Finalmente, observando los espectros ${ }^{1} \mathrm{H}-\mathrm{RMN}$ y ${ }^{13} \mathrm{C}-\mathrm{RMN}$ del compuesto 4 se observa la presencia de un sistema aromático para sustituido, un sistema $\mathrm{CH}_{2} \mathrm{CH}_{2} \mathrm{NH}$ y un grupo acetilo. El sistema de la $\mathrm{N}$-acetil tiramina coincide con las correlaciones observadas en el $\mathrm{H}, \mathrm{H}-\mathrm{COSY}$ y el gHMBC. Además de esto se observan diez señales en el espectro ${ }^{13} \mathrm{C}-\mathrm{RMN}$, dos dobles enlaces trisustituidos, tres metilos alílicos, un alcohol alílico y dos metilenos alílicos. Estos datos concuerdan con la estructura del geranilo enlazado al oxígeno de la tiramina y de configuración $E$, como lo corrobora el cambio químico a $\delta 16.2$ q de C-9`(Kalinowski 1984).

Debido a que los demás compuestos aislados (Fig. 5) ya han sido previamente descritos no se discute su elucidación estructural.

El re-estudio de la planta Amyris brenesii, revela al comparar con los metabolitos obtenidos en estudios previos de esta planta, que especímenes morfológicamente idénticos, poseen compuestos estructuralmente diferentes, lo cual sugiere que los factores geográficos y climáticos son importantes en determinar los tipos de metabolitos presentes en las plantas. 
La determinación de las causas de tales diferencias, escapa de los objetivos del presente trabajo.

\section{AGRADECIMIENTOS}

A la fundación Volkswagen por el apoyo económico en la realización de este proyecto.

\section{RESUMEN}

El estudio fitoquímico de las partes aéreas de Amyris brenesii (Rutaceae) recolectadas en Río Cuarto, Grecia, Alajuela (Costa Rica) mostró la presencia de cuatro nuevos compuestos: la 6-hidroxi-6-O-(3-hidroximetil-3-metilalil)angelicina 1, el 6-(N-acetil-2-etanamin)-2,2-dimetil-2Hcromeno 2, el lignano 2,5-deshidrohinokinina 3 y la $\mathrm{N}$-acetil-O-(geranil)-tiramina 4. Adicionalmente se aislaron los lignanos hinokinina 5, y justicidina $\mathrm{E}$ 6, las cumarinas escopoletina 7 y marmesina 8, el ácido 24-moretenoico 9 y el O-(3,3-dimetilalil)-halfordinol 10. Las separaciones se llevaron a cabo mediante la aplicación de técnicas cromatográficas y la elucidación de las estructuras se realizó con la ayuda de técnicas espectroscópicas de Resonancia Magnética Nuclear (RMN) de una y dos dimensiones.

Palabras Clave: Rutaceae, Amyris, brenesii, lignanos, furocumarina, cromona.

\section{REFERENCIAS}

Basil, A.B. \& H. Parkins. 1978. Amyris of Jamaica. Nicotinamides of Amyris plumieri. Tetrahedron Lett. 30: 2723-2726.

Cordova, H.E. \& L.E. Garelli. 1974. A new coumarin in Amyris simplicifolia. Phytochemistry 13: 758-760.

Domínguez, X.A., G. Cano, I. Luna \& A. Dieck. 1977. Two coumarins from the aereal parts of Amyris madrensis. Phytochemistry 16: 1090.

Dominguez, X.A., R. Franco, G. Cano, S. García, A. Dieck, A. Celia, A. Pedroza \& I. Martínez. 1985. Three Pyranocoumarins from Amyris marshaii standl. Rev. Latinoamer. Quim. 16: 52-53.

Hasbun-Pacheco, C.\&M.Calderón. 1990.2,5-Diaryloxazols and coumarins from roots of Amyris brenesii. Fitoterapia 61: 88 .

Hasbun-Pacheco, C. \& O. Castro-Castillo. 1986. Coumarins from bark of Amyris barbata. J. Nat. Prod. 49: 948-949.
Hasbun-Pacheco, C. \& O. Castro-Castillo. 1988. Ocurrence of coumarins and amides in the bark of three Amyris species from Costa Rica. Spect. Intern. J. 6: 181184.

Hasbun-Pacheco, C. \& O. Castro-Castillo. 1988. A new benzamide from bark of Amyris brenesii. J. Nat. Prod. 51: $817-818$.

Holdridge, L.R. \& L.J. Poveda. 1975. Árboles de Costa Rica, Centro Científico Tropical, San José, Costa Rica.

Hwang, B.Y., H.B. Chai, B.S. Leonardus, S. Riswan, N.R. Farnsworth, G.A. Cordell, J.M. Pezzuto \& A.D. Kinghorn. 2003. Cytotoxic triterpens from the twigs of Celtis phiplipinensis. Phytochemistry 62: 197-201.

Kalinowski, H.O., S. Berger \& S. Braun. 1984. ${ }^{13} C$ NMR Spektroskopie. Georg Thieme Stuttgart, Alemania.

Kato, M.J., M. Yoshida \& O.R. Gottlieb. 1990. Lignoids and arylalkanones from fruit of Virola elongata. Phytochemistry 29: 1799-810.

Murillo, R. \& J. Jakupovic. 2000. Clerodanos y secoclerodanos de Croton jimenezii. Ing. Cienc. Quim. 19:68.

Seida, A. 1979. A Isolation, identification and structure elucidation of cytotoxic and antitumor principles from Ailantus integrifolia, Amyris pinnata and Balanites aegyptiaca. Diss. Abstr. Int. (Sci). 39: 4843.

Shen, C.C., C.L. Ni, Y.L. Huang, R.L. Huang \& C.C. Chen. 2004. Furanolabdane diterpenes from Hypoestes purpurea. J. Nat. Prod. 67: 1947-194

Subbaraju, G.V. \& K.R. Pillai. 1996. Lignans from Justicia diffusa Willd. Indian J. Chem. 35: 1233-1234.

Tada, T. \& T. Nakatsuka. 1968. Wood extractives of Asunaro. Mokuzai Gakkaishi. 14: 344.

Therien, M., B.J. Fitzsimmons, J. Scheigetz, D. Macdonald, L.Y. Choo, J. Guay, J.P. Falgueyret \& D. Riendeau. 1993. Justicidin E: a new leukotriene biosynthesis inhibitor. Bioorg. Med. Chem. Lett. 3: 2063-2066.

\section{REFERENCIAS DE INTERNET}

Instituto Nacional de Biodiversidad INBio. 2005. (Consultado: Junio, 2005; http://www.inbio.ac.cr/ bims/k03/p13/c045/o0133/f01656.htm). 
